\title{
Uma Introdução aos Delineamentos Experimentais de Sujeito Único
}

\author{
Angelo Augusto Silva Sampaio \\ Flávia Henriques Baião de Azevedo \\ Luciana Roberta Donola Cardoso \\ Camila de Lima \\ Mateus Brasileiro Reis Pereira \\ Maria Amalia Pie Abib Andery
}

Pontifícia Universidade Católica de São Paulo

\begin{abstract}
RESUMO
Os delineamentos de sujeito único constituem a abordagem experimental preferida por muitos pesquisadores do comportamento. Com o objetivo de introduzir o tema, discute-se o que é experimentação, definem-se variáveis independentes, dependentes e estranhas e apresentam-se as etapas de um experimento. A definição de delineamento experimental é detalhada, destacando-se suas duas estratégias mais difundidas: delineamentos entre-grupos (ou entre-sujeitos) e delineamentos de sujeito único (ou intra-sujeitos). A partir das diferenças básicas entre essas duas estratégias, apontamse as principais características dos delineamentos experimentais de sujeito único enfatizando a estratégia dos estados estáveis. Os delineamentos experimentais de sujeito único mais comumente utilizados são apresentados e exemplificados (delineamento de reversão, linha de base múltipla, mudança de critério, de sonda e de retirada), ressaltando suas possíveis vantagens e desvantagens. Por fim, são destacadas as diversas possibilidades de combinação de delineamentos.
\end{abstract}

Palavras-chave: experimentação; delineamentos experimentais de sujeito único; delineamento de reversão; delineamento de linha de base múltipla; delineamento de mudança de critério.

\begin{abstract}
An introduction to Single-Subject Experimental Designs

Single-subject designs are the experimental designs of choice for many behavior researchers. The purpose of the present article is to present the basic features of single-subject designs. In order to do so, the related concepts of experimentation, independent, dependent, and extraneous variables and the stages that comprise an experiment are introduced. A definition of experimental design is presented and the two most common research strategies are described: group (or between-subjects) designs and single-subject designs. The basic differences between the two strategies are presented. The main characteristics of single-subject designs and the most common single-subject designs are presented with emphasis on the strategy of steady states. Reverse designs, multiple baseline designs, changing criterion designs, and probe designs are described, as well as their strengths and weaknesses. Finally, the possibility of mixed experimental designs is also discussed.
\end{abstract}

Keywords: experimentation; single-subject designs; reversal design; multiple baseline design; changing criterion design.

Conduzir um experimento é uma tarefa complexa composta de diversas etapas. Uma dessas etapas é o delineamento experimental. Pelo menos dois grandes tipos de delineamento experimental convivem na ciência: delineamentos entre-grupos e de sujeito único. Neste artigo serão apresentadas e discutidas algumas das características mais importantes do método experimental de sujeito único, destacando-se seus delineamentos mais usuais. A ênfase será na aplicação de tal método a pesquisas sobre o comportamento dos organismos.

\section{0 que é experimentação}

A experimentação é um modo fundamental de produzir conhecimento no mundo contemporâneo. Mas o que é um experimento? De modo geral, 
Um experimento é uma série de ações que resulta em um conjunto de observações especiais que não seriam possiveis de outra forma. A experimentação é um modo de simplificar as condições sob as quais a observação é feita de modo que um fenômeno possa ser observado mais claramente (Johnston \& Pennypacker, 1993a, pp. 8-9, destaque no original).

Isaac Newton, por exemplo, em seu experimento clássico sobre a decomposição da luz, permitiu a entrada em um quarto escuro, por um furo na veneziana da janela, de um pequeno raio de sol (Crease, 2006). Newton organizou o quarto de modo que esse raio incidisse sobre um prisma de vidro; ao atravessar o prisma, o raio se decompôs, projetando sobre um anteparo à sua frente todas as cores do arco-íris. Newton, além disso, havia feito um furo nesse anteparo, de modo que podia projetar sobre esse furo apenas uma das cores produzidas. $\mathrm{O}$ raio de luz colorida que atravessava esse furo, por sua vez, atingia um segundo prisma desviando-se da sua rota original (isto é, refratava-se) antes de atingir a parede sem se decompor ou mudar de qualquer outro modo. A partir desse experimento (e de outros similares) Newton pôde comprovar que a luz branca não era "pura" como se pensava na época, mas composta por todas as cores do arco-íris, estas sim "puras".

Além de produzir uma observação que seria muito difícil (ou impossível) de se obter cotidianamente (uma "observação especial"), o experimento de Newton, ou ainda, o modo como Newton simplificou as condições de observação ao organizar seu quarto com os prismas e o anteparo, permitiu-lhe:

- estabelecer uma relação causal ${ }^{1}$ entre a cor dos raios de luz que incidiam sobre o segundo prisma e o ângulo em que estes eram refratados: o "tamanho do desvio" quando os raios de luz eram refratados (sua refringência) era uma propriedade dos próprios raios de luz, e não dos prismas;

- compreender diversos fenômenos naturais relacionados (por exemplo, a formação do arco-íris) e

- contribuir para várias aplicações práticas (por exemplo, a construção de melhores telescópios).

Estes pontos ilustram três objetivos e pontos fortes da experimentação: o estabelecimento de relações causais, a compreensão de fenômenos e a promoção de aplicações práticas.

\section{Variáveis independente, dependente e estranha}

O objetivo essencial de um experimento é estabelecer relações causais entre eventos, ou melhor, relações sistemáticas entre variáveis - aspectos dos fenômenos que podem assumir qualquer valor de um determinado conjunto (o sexo de uma pessoa, por exemplo, é uma variável, já que pode assumir pelo menos dois valores: masculino ou feminino). $\mathrm{Na}$ experimentação, dividem-se estas variáveis em pelo menos duas categorias principais: as variáveis experimentais ou independentes (VIs) e as variáveis observadas ou dependentes (VDs) ${ }^{2}$. As variáveis independentes são aquelas manipuladas pelo pesquisador, que está interessado em seus efeitos. As variáveis dependentes, por sua vez, são aspectos/dimensões do fenômeno de interesse, são as variáveis que o pesquisador mede em busca dos efeitos das VIs. Aqui, vê-se uma característica fundamental dos experimentos: a manipulação de variáveis. No exemplo da decomposição da luz, Newton precisou planejar um quarto escuro no qual apenas um pequeno orifício permitia a entrada de luz solar e precisou posicionar adequadamente os prismas e o anteparo (todos constituindo VIs), de modo a possibilitar suas observações sobre as mudanças na luz solar (a VD). Sem tais manipulações, ele não teria chegado às suas conclusões, fundamentais para o desenvolvimento da óptica.

Para estabelecer com clareza a relação entre VIs e VDs, o controle experimental é imprescindível. É necessário o manejo adequado tanto das VIs quanto das VDs e de todos os fatores que possam interferir nos efeitos das primeiras sobre as últimas - tais fatores (eles mesmos mais variáveis) são chamados de variáveis estranhas (VEs) (Johnston \& Pennypacker, 1993a). Em um estudo sobre o tratamento da depressão, por exemplo, as conclusões acerca da eficácia de um novo medicamento (VI) sobre comportamentos considerados depressivos (VDs) deveriam levar em conta a possibilidade de que outras variáveis, como freqüentar uma psicoterapia e fazer exercícios físicos (VEs), possam afetar os resultados.

Portanto, a tarefa que um cientista se propõe ao fazer um experimento exige cuidados e planejamento. A partir de suposições sobre as variáveis que constituem um fenômeno de seu interesse (VDs), o experimentador deve planejar como medir esse fenômeno, como manipular as condições que têm função em sua determinação (VIs) e como diminuir ou, pelo menos, isolar os efeitos de outras condições que podem afetar o fenômeno de interesse, mas que não se pode ou não se pretende manipular (VEs). 


\section{As etapas de um experimento}

A realização de um experimento é um processo complexo no qual o experimentador percorre várias etapas interrelacionadas. Estas etapas não devem ser entendidas como momentos predeterminados envolvendo uma sequência rígida de passos, mas como uma divisão pedagógica de um conjunto de ações fluido e flexível.

A partir da definição de uma pergunta experimental (o que se busca aprender com o experimento), o experimentador deve considerar: 1) como o(s) fenômeno(s) em estudo será(ão) medido(s), garantindo a validade e a confiabilidade dessas medidas; 2) como as condições experimental e controle serão arranjadas para permitir comparações entre elas (delineamento experimental); 3) como os dados (as medidas obtidas) serão analisados, e 4) que conclusões são possíveis a partir dos resultados.

Nenhuma dessas etapas pode ser compreendida isoladamente, já que todas as decisões tomadas pelo pesquisador ao longo do planejamento e execução de um experimento dependem umas das outras. Apesar da interdependência das etapas de um experimento, o que se pretende aqui é possibilitar uma visão geral da fase de delineamento experimental.

\section{Delineamento experimental}

Todo experimento envolve duas condições básicas: condição controle (ou linha de base) e condição experimental. A condição controle é aquela na qual a VI não está presente. Ela permite avaliar os efeitos de todas as variáveis, que não a VI, sobre a VD. A condição experimental, por sua vez, é aquela na qual a VI está presente. Assim, é necessário que haja no experimento pelo menos duas condições que difiram apenas quanto à presença da VI. Mantendo todas as variáveis constantes entre as duas condições e variando apenas a VI, o experimentador está em posição favorável para concluir se as possíveis mudanças medidas são frutos de sua manipulação.

Segundo Johnston e Pennypacker (1993a), delinear um experimento é planejar condições controle e experimentais de modo a permitir comparações significativas entre elas, verificar os efeitos da VI e responder ao problema de pesquisa. Por esta razão, é extremamente importante que o experimentador conheça as características, as possibilidades (os pontos fortes) e os limites (os pontos fracos) dos delineamentos experimentais em geral, e dos delineamentos experimentais que planeja.
Dito de outra forma, delinear um experimento é decidir sobre: 1) quantos sujeitos ${ }^{3}$ serão utilizados; 2) quantas serão as condições (controle e experimental) utilizadas; 3) quando, por quanto tempo e em que ordem as condições serão introduzidas; e 4) quando e quantas vezes as medidas de interesse serão realizadas.

\section{Delineamentos entre-grupos e delineamentos de sujeito único}

Dois principais tipos de delineamentos experimentais são os de sujeito único (ou intra-sujeito) e os delineamentos entre-grupos (ou entre-sujeitos). A abordagem mais tradicionalmente utilizada na Psicologia e nas Ciências Sociais é a que emprega delineamentos entre-grupos. Nestes delineamentos os efeitos de uma condição experimental são avaliados pela comparação entre diferentes grupos de sujeitos, submetidos, cada um dos grupos, a diferentes condições. Nos delineamentos entre-grupos cada sujeito é exposto a apenas uma das condições do experimento. Além disso, todos os sujeitos de um grupo são expostos às condições por um mesmo período de tempo. Finalmente, as medidas de interesse costumam ser realizadas poucas vezes para cada sujeito e, de modo geral, envolvem o agrupamento dos dados relativos aos sujeitos que compõem cada grupo (por exemplo, pela obtenção de médias, desvios padrão, ou porcentagens de sujeitos que atingem certo critério). A comparação entre os resultados dos grupos, por sua vez, muitas vezes envolve o uso de instrumentos da estatística inferencial, como testes para avaliar a fidedignidade estatística dos dados e a significação das diferenças encontradas entre os grupos (Johnston \& Pennypacker, 1993a, 1993b; Kerlinger, 1973; Kidder, 1987). Em um exemplo bem simples, um novo medicamento poderia ser testado pela distribuição aleatória de sujeitos em dois grupos. Um grupo recebe o medicamento (condição experimental) e o outro grupo recebe apenas um placebo (condição controle). Os sintomas da doença-alvo são medidos apenas duas vezes: antes e depois dos respectivos tratamentos. Os resultados obtidos em cada grupo são então estatisticamente comparados entre si para avaliar a eficácia do medicamento. Se os sintomas tiverem uma redução estatisticamente significativa no grupo que recebeu o medicamento, a redução poderia ser atribuída ao medicamento.

Outra abordagem experimental com uma longa tradição na história das ciências é o delineamento de 
sujeito único. Sua utilização no estudo do comportamento humano tem sido defendida por diversos pesquisadores (Estes \& Skinner, 1941; Ferster \& Skinner, 1957; Johnston \& Pennypacker, 1993a, 1993b; Kazdin, 1982; Matos, 1990; Sidman, 1960/1976; Skinner, 1938, 1947, 1956). Entre os argumentos a apoiar sua utilização destaca-se o fato do comportamento ser um fenômeno característico de organismos individuais, que interagem de maneira única com o mundo - dois indivíduos nunca se comportam da mesma maneira. Argumenta-se também que cálculos que agregam resultados, como médias de desempenhos de grupos de indivíduos, não representam corretamente o desempenho de nenhum dos seus membros, pois raramente um sujeito se comporta exatamente como essa média. Além disso, agregar resultados dessa forma envolve misturar dados efetivamente comportamentais (relativos aos desempenhos dos sujeitos) com a diferença entre os desempenhos (de dois ou mais sujeitos) que é um dado não comportamental, o que não é útil na explicação do comportamento de um organismo singular.

Os delineamentos de sujeito único têm como característica principal tratar os sujeitos individualmente, tanto no que se refere às decisões relativas ao próprio delineamento, quanto ao processamento dos dados - o que não implica a utilização de um único sujeito por experimento. Neste modelo de delineamento, os sujeitos são expostos a uma série de condições, mensurando-se repetidamente o desempenho do organismo e verificando-se se há uma relação ordenada entre as condições manipuladas no experimento e as alterações nessas medidas (Matos, 1990). Diferentemente dos delineamentos entre-grupos, um mesmo sujeito é submetido a todas as condições do experimento e as observações são realizadas de forma contínua no decorrer de todo o processo.

\section{A estratégia dos estados estáveis}

Arranjar comparações entre as condições em um experimento conduzido com um delineamento de sujeito único não é uma tarefa simples. A precisão e generalidade das conclusões dependem da qualidade das medidas do desempenho do sujeito ${ }^{4}$.

Caso qualquer coisa no desempenho do sujeito, seja na condição controle, seja nas condições experimentais, não for típica dos efeitos usuais destas condições, então 'subtrair' o desempenho na condição controle do responder sob a condição experimental resul- tará em uma diferença que é de algum modo enganadora (Johnston \& Pennypacker, 1993a, p. 197).

Ou seja, inferir efeitos da variável independente (VI) apenas pelas diferenças nas medidas da variável dependente (VD) de uma condição para a outra, sem se ter conhecimento suficiente sobre os resultados de medida que seriam típicos em cada uma das condições pode levar ao erro. Além disso, a precisão e a generalidade dos resultados de um experimento com delineamento de sujeito único dependem fortemente do controle sobre as variáveis estranhas (VEs) e qualquer conclusão sobre os resultados precisará considerar e trabalhar com a influência destas variáveis. Um recurso central para se lidar com tais dificuldades quando se trabalha com delineamento de sujeito único é a estratégia dos estados estáveis. Essa estratégia envolve medir repetidamente a VD sob condições que são mantidas constantes até se obter dados relativamente estáveis. Estabilidade é definida como um padrão que mostra pouca variação ao longo de um certo período de tempo.

Nos delineamentos entre-grupos, comparam-se os dados agregados de todos os participantes de um grupo com os dados agregados dos participantes de outro grupo e, então, supõe-se que eventuais inconsistências ou variações não esperadas sejam compensadas entre os sujeitos, de modo que os resultados do grupo todo expressariam o resultado típico independente de (descontadas as) variações individuais.

Nos delineamentos de sujeito único essa suposição não pode ser feita. A estabilidade das medidas da VD é um requisito indispensável, uma vez que se compara em cada sujeito os valores da VD em diferentes condições. Se não há estabilidade em cada condição nada se pode afirmar quando se comparam valores em distintas condições para o mesmo sujeito.

No entanto, estabilidade não pode ser confundida com imutabilidade. Especialmente quando se estuda comportamento há que se esperar contínua variação. Estabilidade dos dados coletados em um experimento no qual se utiliza um delineamento de sujeito único significa, então, que os valores da VD oscilam de certa maneira (com certo padrão) que o experimentador considera estável. Não se deve esperar também que necessariamente os valores de uma VD mudem imediata ou abruptamente quando uma VI é retirada ou introduzida. Especialmente no estudo do comportamento, ao manipular condições ambientais freqüentemente se está diante de um processo no qual a mudan- 
ça na relação entre sujeito e ambiente gradativamente estabelece um novo padrão comportamental. Como conseqüência, nos estudos que utilizam o método do sujeito único atribui-se grande importância à estabilidade, ao mesmo tempo em que se reconhece que não há critérios universalmente bons de estabilidade. Por essas razões também, em cada condição de um experimento múltiplas medidas da VD são necessárias. É preciso efetuar seguidas medidas em cada condição, até que o resultado dessas medidas atinja algum critério de estabilidade; só então o experimentador pode, com alguma segurança, manipular a condição. Finalmente, outra implicação da chamada estratégia de estados estáveis é que a duração e muitas vezes a própria seqüência de condições do experimento dependem do desempenho das medidas da VD (Johnston \& Pennypacker, 1993a, 1993b; Sidman, 1960/1976).

A estabilidade nas medidas da VD em cada condição de um experimento com delineamento de sujeito único torna-se, então, critério necessário para se decidir pela continuidade ou mudança de condições em cada momento do experimento. Espera-se que o próprio resultado das manipulações e medidas seja o "árbitro" das decisões quanto ao delineamento, como, por exemplo, quando introduzir a condição experimental.

Três razões tornam a estratégia dos estados estáveis e os delineamentos de sujeito único especialmente proveitosos. Em primeiro lugar, a sistemática manipulação de condições para cada sujeito experimental e a estratégia dos estados estáveis permite que as relações entre VI e VD sejam inferidas a partir das sistemáticas mudanças mensuradas nas VDs correlacionadas com mudanças nas condições dasVIs. O experimentador pode, assim, identificar características típicas da VD, sugerindo o modo como a VD está relacionada às suas variáveis controladoras (VIs e/ou VEs) e problemas no processo de mensuração.

Em segundo lugar, os delineamentos de sujeito único e a estratégia dos estados estáveis permitem avaliar o grau de controle experimental obtido, ou seja, quanto o pesquisador pode ter certeza de que a VI é responsável pelas mudanças observadas na VD.

Em terceiro lugar, a comparação dos desempenhos da VD em várias condições experimentais, auxilia a afirmação de representatividade (os dados obtidos de fato correspondem aos efeitos principais da condição avaliada?) e generalidade (os dados obtidos são replicáveis?) dos resultados em cada condição.
Todas essas características dos delineamentos experimentais de sujeito único podem ser alcançadas por uma gama ilimitada de arranjos de condições do experimento. Algumas das opções mais simples e comumente utilizadas serão brevemente apresentadas e exemplificadas a seguir.

Desde já é preciso ressaltar que os delineamentos de sujeito único não se resumem àqueles que serão aqui descritos. O cientista planeja seu experimento de maneira a responder suas perguntas de pesquisa com alguma convicção de que o seu controle experimental deu origem a resultados que têm algum grau de precisão, validade e generalidade. Experimentos conduzidos com delineamento de sujeito único são planejados de modo a ser afetados pelas características do fenômeno em estudo e pelos resultados obtidos já durante sua execução. Como implicação, não há um limite preestabelecido para as possibilidades de inovação experimental (Sidman, 1960/1976). No entanto, algumas estratégias de delineamento experimental têm sido freqüentemente utilizadas, têm-se demonstrado úteis e serão apresentadas aqui.

\section{Delineamento $A B A B$ ou de reversão}

A lógica do delineamento $\mathrm{ABAB}$ (onde $\mathrm{A}=$ condição controle e $\mathrm{B}=$ condição experimental) consiste em realizar sucessivas comparações entre condições controle e condições experimentais em um mesmo experimento (sendo desejáveis no mínimo três condições). Ou seja, com este delineamento busca-se demonstrar os efeitos de uma VI pela retirada de uma condição e pela reintrodução de outra das condições já apresentadas (reversão à condição anterior). Por isso, tais delineamentos são também chamados de delineamentos de reversão.

Nos delineamentos de reversão cada nova condição proporciona uma nova oportunidade para comparar o desempenho da VD e testar se tal desempenho é alterado com a introdução e retirada da VI (Kazdin, 1982). Os resultados deste tipo de delineamento podem sugerir que a VI foi de fato responsável pela modificação na VD se o desempenho da VD se modificar quando a condição experimental for introduzida (B), voltar para o nível anterior da condição controle (ou próximo a ele) quando a condição experimental for retirada (A) e mudar novamente quando a VI for reapresentada (B).

Para exemplificar este delineamento, consideremse os dados apresentados por Miller e Kelley (1994), 
que investigaram os efeitos de um procedimento de "estabelecimento de metas e contrato comportamental" sobre o desempenho nas lições de casa de uma criança de 10 anos (Richard). Para tanto, o procedimento se iniciou com a simples observação do comportamento-alvo (tempo gasto nas lições), sem nenhuma interferência programada (condição controle = A). A seguir, introduziu-se a condição experimental (B), na qual a criança era recompensada ao atingir metas estabelecidas junto com seus pais para a realização de cada lição de casa designada pela escola. Uma fase de reversão à condição controle (A) foi, então, introduzida e, por fim, retornou-se a uma nova condição experimental (B). Na Figura 1 representa-se o tempo (porcentagem de intervalos) pelo qual Richard permaneceu trabalhando em suas lições nos dias em que a condição controle (A) e a condição experimental (B) estiveram em vigor. As mudanças sistemáticas no desempenho de Richard (representadas na Figura 1), quando o contrato foi introduzido e, especialmente, quando foi retirado e novamente reintroduzido sugeriram a Miller e Kelley que o contrato (VI) foi variável significativa sobre o fazer lição de casa (VD) de Richard.

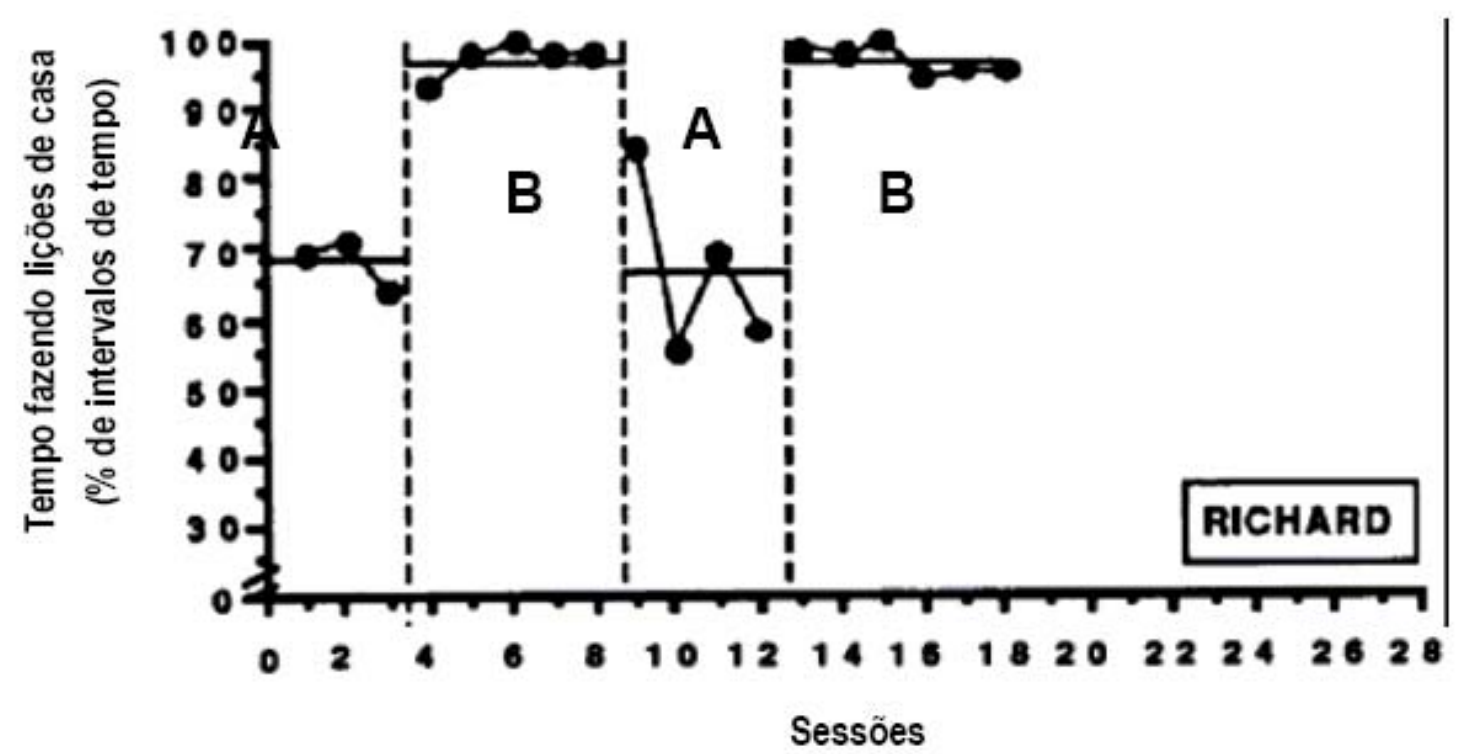

Figura 1. Um exemplo de delineamento de reversão (figura adaptada de Miller \& Kelley, 1994). As linhas pontilhadas indicam mudanças nas condições do experimento: $A$ = condição controle, $B$ = condição experimental.

Como Kazdin (1982) salientou, os delineamentos $\mathrm{ABAB}$ não se restringem a quatro condições em uma mesma seqüência (como o rótulo sugere). Sempre que se alternam condição controle e condição experimental trata-se de um delineamento de reversão ou ABAB. Outras variações são possíveis e freqüentemente encontradas na literatura. Entre tais possibilidades destaca-se a inversão da ordem das condições (por exemplo, delineamentos BABA). Tal inversão é muitas vezes necessária em pesquisas aplicadas quando o comportamento (a VD) a ser modificado apresenta características de risco, ou quando só ocorre raramente. Outra possibilidade é a alteração do número de condições (por exemplo, ABABABAB), o que é especialmente importante quando se pretende ter maior clareza sobre os efeitos produzidos em cada condição e sua relação com a variável manipulada. Por fim, pode-se incluir um número maior de condições experimentais ou diferentes VIs. Nesse caso, diferentes letras rotulam distintas manipulações das VIs (por exemplo, ABCADABCD).

Há alguns problemas que podem afetar o delineamento $\mathrm{ABAB}$ e suas variações, tornando-o indesejável ou impossível de ser utilizado. O primeiro problema é a não-reversão das medidas da VD. Caso haja possibilidade da VD não ser revertida para os níveis da condição controle quando a VI for retirada, então o delineamento de reversão não é adequado. Na avaliação de um procedimento de ensino (VI) para a aquisição de leitura (VD), por exemplo, não se deveria espe- 
rar que os participantes deixassem de ler após o término do procedimento - e, conseqüentemente, não se deveria utilizar o delineamento ABAB. Por outro lado, se um delineamento de reversão foi utilizado e não se observou a reversão dos valores da VD quando se retirou a VI, pode-se estar diante de um problema de controle experimental. Ou seja, variáveis não controladas no experimento (VEs) seriam responsáveis pelos resultados, o que não invalidaria o delineamento utilizado se tal controle puder ser estabelecido. Finalmente, em pesquisas aplicadas, a característica central desse delineamento pode trazer um problema quando a VD envolver comportamentos cuja reversão não é desejável, por exemplo, por razões éticas. Na avaliação de um procedimento específico (VI) para reduzir a freqüência de comportamentos auto-lesivos (VD), a reversão à condição controle pode levar a um aumento na freqüência dos comportamentos, o que é eticamente inaceitável.

\section{Delineamento de linha de base múltipla}

No delineamento de linha de base múltipla, mais de uma VD é mensurada e analisada ao mesmo tempo, são estabelecidas, portanto, mais de uma linha de base (condições controle), e as VIs são introduzidas em momentos seguidos (distintos) no tempo para cada uma delas. Nos estudos comportamentais essas VDs podem ser: duas ou mais respostas emitidas por um mesmo sujeito ou uma mesma resposta emitida por dois ou mais sujeitos (delineamentos de linha de base múltipla entre respostas ou comportamentos ${ }^{5}$ ), ou uma mesma resposta emitida por um sujeito em duas ou mais situações ou ambientes físicos/sociais (delineamento de linha de base múltipla entre ambientes ou settings) (Kazdin, 1982).

O delineamento é implementado da seguinte forma: após todas as medidas de VDs atingirem estabilidade na condição controle, a primeira condição experimental é introduzida, manipulando-se a VI em relação a uma primeira VD selecionada. Quando as medidas desta primeira VD atingem estabilidade na condição experimental e, caso as demais tenham se mantido estáveis na condição controle, manipula-se a VI em relação a uma segunda $\mathrm{VD}$, mantendo-se a primeira na condição experimental e as demais na condição controle. Novamente, espera-se que a segunda VD atinja estabilidade para aplicar a VI em uma terceira VD, mantendo a aplicação da VI nas anteriores. Assim, segue o procedimento, até aplicar-se a VI à última VD. Durante todo o procedimento, são registrados os dados de todas as VDs na condição controle e na condição experimental.

Como um exemplo deste delineamento entre comportamentos emitidos por um mesmo indivíduo, Borstein, Bellack e Hersen (1977) trabalharam com crianças consideradas não assertivas. Depois de avaliar vários comportamentos verbais e não-verbais considerados importantes em interações sociais (VDs), promoveram treinos de habilidades sociais (VI) para cada um dos comportamentos que eram emitidos em baixa freqüência pelas quatro crianças dessa pesquisa. Os dados foram coletados em sessões em que a criança se engajava em uma interação com um parceiro. Na Figura 2 são apresentados os resultados relativos a três comportamentos que foram alvo de intervenção para uma das crianças (Jane). Neste caso, os comportamentos-alvo foram: estabelecimento de contato visual com um parceiro durante uma conversa, que foi medido como a proporção de tempo de interação em que a criança manteve contato visual com o parceiro; falar em tom de voz audível, que foi medido em uma escala de volume de 1 a 5 por observadores; e solicitar que o parceiro mudasse seu comportamento quando inadequado, medido pelo número de solicitações. Após medir as VDs durante a condição controle, aplicou-se o treino de habilidades sociais (VI) para a primeira VD (contato visual). A taxa de contato visual aumentou, mas os outros comportamentos mensurados permaneceram em baixa freqüência. Quando esta primeira VD atingiu estabilidade durante a condição experimental, aplicou-se o procedimento de treino (VI) ao falar em tom de voz audível (a segunda VD). Essa segunda VD sofreu efeito do treino (a avaliação de volume aumentou), e, uma vez estabilizado o comportamento, novamente a VI foi aplicada, agora sobre o terceiro comportamento (VD), que era solicitar mudança de comportamento do outro. 


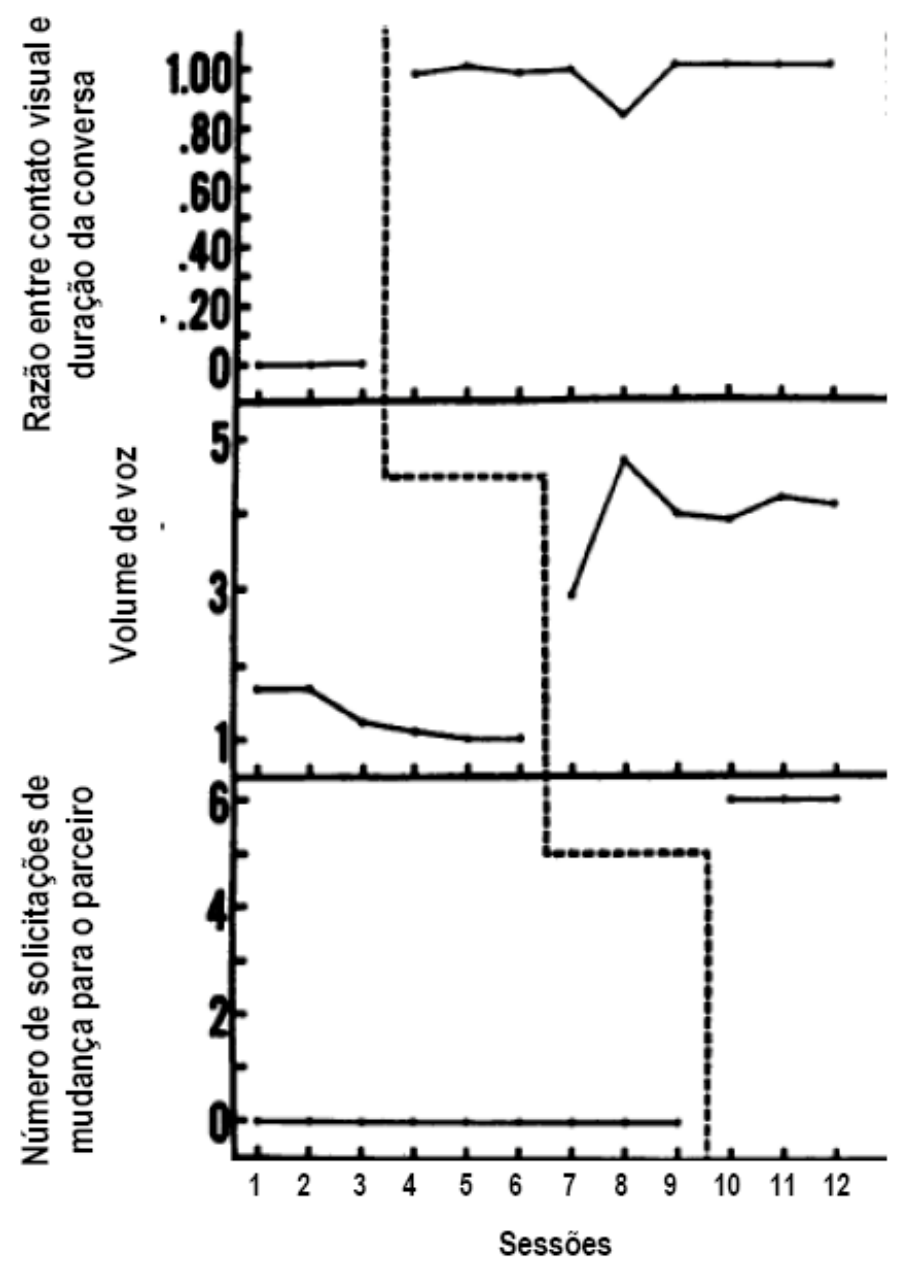

Figura 2. Um exemplo de delineamento de linha de base múltipla entre comportamentos (figura adaptada de Bornstein, Bellack \& Hersen, 1977). Cada quadro indica um comportamento e a linha pontilhada indica a introdução da variável independente.

Em um delineamento com uma única condição controle que é seguidamente reapresentada (ABAB, por exemplo), uma VE pode alterar a VD no exato momento em que é introduzida a VI, o que geraria conclusões imprecisas a respeito do que produziu tal alteração. O delineamento de linha de base múltipla diminui a possibilidade deste tipo de imprecisão. Ele permite que se teste se de fato a VI é responsável por mudanças na VD, já que mudanças semelhantes nos desempenhos das várias VDs quando a VI é seguidamente introduzida refutariam ou, pelo menos, enfraqueceriam a hipótese da influência de VEs nessas mudanças. Também, ter mais de uma resposta, situação ou sujeito em condição controle enquanto outros estão sob o efeito da VI possibilita a avaliação de quais mudanças nessas VDs seriam ou não efeito da VI.
Além disso, nos delineamentos de linha de base múltipla não é necessária a reversão para demonstrar os efeitos da condição experimental. Especialmente em pesquisas aplicadas, este delineamento pode ter um benefício adicional: se a introdução de uma VI para a produção de mudanças na VD for relevante, a introdução da VI em seguidas VDs poderia produzir mudanças efetivas que se estenderiam para outras VDs. Outra vantagem dos delineamentos de linha de base múltipla, principalmente em situações de pesquisa aplicada, é a possibilidade de introdução da VI de maneira mais gradual, permitindo aos experimentadores avaliar criteriosamente os efeitos dos procedimentos empregados, bem como sua execução.

Um dos possíveis problemas com os delineamentos de linha de base múltipla é a possibilidade de interde- 
pendência entre as condições controle. Fala-se em interdependência entre as condições controle quando no momento em que se introduz uma VI sobre uma das VDs ocorre também alteração em outra VD sobre a qual não foi realizada diretamente a manipulação. Neste caso, pode existir uma variável estranha (VE) provocando a mudança na medida da segunda VD, ou, de fato, a VI de alguma maneira tem efeitos mais amplos do que o experimentador esperava. De qualquer modo, o efeito da manipulação, ou melhor, o papel da VI se torna ambíguo. Porém, mais uma vez, especialmente em pesquisas aplicadas, esse efeito pode ser positivo para os participantes. Assim, muitos planos de pesquisa podem prever um delineamento de linha de base múltipla na expectativa de testar se VDs que não são selecionadas para condição experimental, pelo menos no início de um experimento, podem ser alteradas, em condição controle, pela introdução de uma VI sobre outras VDs.

Nos delineamentos de linha de base múltipla devem-se evitar condições controle prolongadas, já que nesse caso a introdução da VI sobre as últimas VDs tem mais probabilidade de sofrer a interferência de outros eventos não controlados (VEs). Para evitar esse tipo de problema são comuns variações nos delineamentos de linha de base múltipla em que se reduz o número de VDs (respostas, situações ou sujeitos) ou se introduz a VI simultaneamente sobre mais de uma VD.

Outra dificuldade relacionada com os delineamentos de linha de base múltipla pode advir quando são observados efeitos inconsistentes da manipulação, ou seja, quando os efeitos das seguidas introduções da VI sobre novas VDs variam. Neste caso, o experimentador pode estar diante de manipulações às quais falta controle experimental (VEs podem estar atuando), ou pode haver problemas em relação às decisões experimentais e à condução do experimento: critérios de estabilidade podem ser inconsistentes (as condições do experimento podem estar sendo alteradas com critérios diferentes em cada momento), ou inadequados (as mudanças de condição são feitas prematuramente, por exemplo, e os resultados mostrariam ainda efeitos de transição); efeitos de ordem na introdução da VI sobre as VDs podem ser relevantes; ou a própria manipulação da VI pode ter problemas (o que é especialmente provável em situações aplicadas que freqüentemente envolvem manipulações que dependem de ações humanas e não de equipamento).

\section{Delineamento de mudança de critério}

Os experimentos com delineamento de mudança de critério começam com uma condição controle, após a qual a condição experimental é introduzida. A característica principal desse delineamento é que a condição experimental é constituída de subcondições ou subfa$\operatorname{ses}^{6}$. Cada uma dessas subfases corresponde a um valor da VD estabelecido como critério que indicaria o efeito da VI, ou como critério para a apresentação de condições ambientais que compõem a VI. Assim, o nível de desempenho (o valor da VD) exigido para mudanças na condição do experimento é alterado repetidamente no curso da condição experimental. O efeito da VI é revelado mostrando que a VD muda gradualmente ao longo do processo.

Embora possa se assemelhar aos delineamentos de sujeito único já apresentados, o delineamento de mudança de critério guarda algumas características que o distinguem dos demais: diferentemente dos delineamentos de reversão esse delineamento não requer retirada ou suspensão temporária da VI para que se demonstre seu efeito sobre a VD, e, diferentemente dos delineamentos de linha de base múltipla, a condição experimental restringe-se a uma única VD (nos experimentos comportamentais, uma resposta, ou dimensão de resposta ou relação comportamental).

Segundo Kazdin (1982), uma característica importante do delineamento de mudança de critério é o número de vezes que o critério é mudado na condição experimental. Como neste delineamento espera-se que a exigência de novos valores nas medidas da VD seja seguida pela obtenção desses valores (o que seria indicador dos efeitos da VI), recomenda-se pelo menos duas mudanças de critério (ou subfases). Tal delineamento, então, é indicado naqueles experimentos em que o estabelecimento de apenas um critério para a condição experimental pode tornar difícil mostrar que a manipulação da VI foi responsável pela mudança na VD.

No delineamento de mudança de critério, a estabilidade da VD em determinado critério antes de se introduzir um outro é de grande importância, pois, caso contrário, é difícil avaliar a relação entre a manipulação da VI e o valor da VD. Uma vez obtida a estabilidade em cada subfase, cada uma delas pode ser utilizada pelo pesquisador como condição controle para a seguinte.

Outro ponto a ser considerado nesse delineamento é como decidir sobre a magnitude das mudanças de 
critério. O tempo necessário para que um critério seja atendido pode servir de indicador para o pesquisador: o critério não pode ser tão exigente a ponto de não ser jamais atingido ou de ser atendido muito tardiamente e nem tão pouco exigente a ponto de não ser possível obter mudanças significativas nos patamares de medida da VD. Isso quer dizer que em experimentos com esse delineamento muitas vezes os critérios estabelecidos em cada subfase dependem dos desempenhos dos sujeitos e são decididos no decorrer das manipulações. Tal característica exemplifica uma das vantagens já destacadas dos delineamentos de sujeito único: a possibilidade de ajuste do delineamento a partir do desempenho concreto do sujeito durante a pesquisa.

O experimento de De Luca e Holborn (1992) é um exemplo do emprego desse delineamento. Três garotos obesos e três garotos não obesos foram solicitados a pedalar uma bicicleta ergométrica com a afirmação "exercite-se o quanto você quiser". Na condição controle, os experimentadores mediram o número de voltas pedaladas por minuto em cada sessão, sem programar nenhuma conseqüência para essa atividade. $\mathrm{Na}$ condição experimental, calculou-se o número médio de voltas por minuto durante a condição controle para cada participante e estabeleceu-se como critério para reforçamento um número de voltas aproximadamente $15 \%$ acima desta média. Na condição experimental, pedaladas produziam, então, em um esquema de Ra- zão Variável (VR), cujo valor foi estabelecido como descrito, o acendimento de uma luz e o toque de uma campainha, juntamente com a liberação de um ponto. Os pontos acumulados eram trocados no final da sessão por objetos escolhidos pelos próprios garotos (brinquedos, revistas em quadrinho etc.). O número de pedaladas exigido para se ganhar um ponto foi gradualmente aumentado nas subcondições experimentais. A cada nova subcondição o valor da VR exigida (número médio de pedaladas que produziam conseqüências), foi aumentado em torno de $15 \%$, de modo que ao final do experimento os garotos estavam pedalando muito mais do que na condição controle. (Após três subcondições experimentais, a condição controle foi reintroduzida e, finalmente, o último valor de VR foi retomado para todos os participantes. Essas duas últimas condições não são apresentadas na Figura 3).

$\mathrm{Na}$ Figura 3, o desempenho (número médio de voltas pedaladas por minuto em cada sessão) de um dos participantes (Paul) é representado. Os valores de VR estabelecidos em cada subcondição estão representados pelas linhas tracejadas horizontais: como se pode ver, para ganhar pontos, o participante deveria emitir 80 pedaladas em média na primeira subcondição. Durante as seguidas mudanças de critério a exigência foi gradualmente aumentada até 120 pedaladas em média.

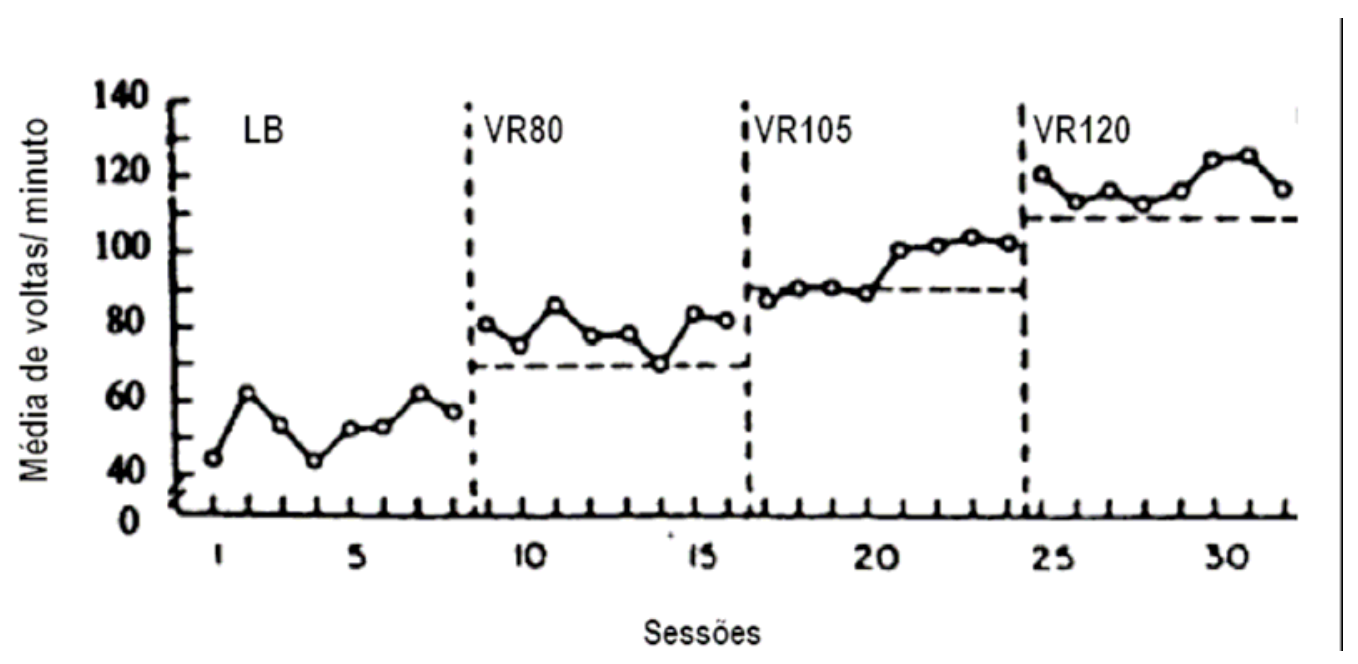

Figura 3. Um exemplo do emprego do delineamento de mudança de critério (figura adaptada de De Luca \& Holborn, 1992). $\mathrm{LB}=$ linha de base ou condição controle.

Neste delineamento a direção das mudanças de critério das subfases pode ser alterada. Geralmente, os efeitos da VI são avaliados examinando-se uma mudança na VD em uma mesma direção ao longo do tempo (mudanças unidirecionais), por exemplo, um aumento ou uma diminuição na freqüência do comportamento que constitui a VD. Entretanto, podem surgir algumas dificuldades na avaliação de mudanças 
unidirecionais ao longo da condição experimental. O desempenho pode melhorar em função de VEs e não da VI e esses efeitos podem ser difíceis de distinguir, a não ser que as mudanças na VD acompanhem estritamente o critério estabelecido em cada subfase. O controle exercido pela VI pode ser melhor detectado alterando-se a direção da mudança de critério: se a exigência de um desempenho da VD em direção oposta à que vinha ocorrendo for seguida de uma mudança correspondente nos valores obtidos, levando a uma mudança bidirecional na VD, aumenta a confiança do experimentador no controle exercido pela VI. Por exemplo, o critério está sendo aumentado ao longo da condição experimental, então, durante uma subfase, ele é diminuído para um nível anterior. Segundo Kazdin (1982), essa subfase constitui uma "mini-fase de reversão". A condição experimental não é suspensa, mas as mudanças esperadas no comportamento são invertidas. Caso tais mudanças de fato aconteçam na direção contrária daquelas da fase anterior fortalece-se a hipótese de que a VI foi responsável pela mudança.

Uma limitação do delineamento de mudança de critério é que quando não há uma correspondência exata entre a mudança de critério e a mudança no desempenho, pode ser difícil avaliar se a VI colabora para a mudança do comportamento.

\section{Delineamentos de sonda}

Os delineamentos de sonda são geralmente utilizados para verificar se outras VDs, que não aquela que foi mensurada nas condições do experimento, foram afetadas pelas manipulações realizadas. Uma sonda, portanto, refere-se a uma avaliação de VDs quando nenhuma condição de VI planejada em relação àquelas VDs está sendo manipulada. Nesses delineamentos, avalia-se a transferência dos efeitos observados sobre uma VD para outras (sejam elas respostas diferentes, ou uma mesma resposta em situações diferentes, por exemplo), realizando medidas (observações) ocasionais de outras VDs que não a VD do experimento (Kazdin, 1982). No estudo de Miller e Kelley (1994), citado anteriormente para ilustrar os delineamentos de reversão, por exemplo, os pesquisadores poderiam ter medido ocasionalmente o tempo pelo qual a criança permanecia trabalhando em tarefas domésticas (ajudar os pais na arrumação da casa, lavar pratos etc.). Estas sondas poderiam ter avaliado se os efeitos do procedimento de contrato comportamental (VI) transferiram-se do desempenho nas lições de casa (VD) para outras atividades domésticas de Richard que não foram diretamente manipuladas (outras VDs). Assim, uma qualidade importante destes delineamentos é que eles são um meio econômico de avaliar a generalidade dos efeitos das manipulações experimentais.

\section{Delineamentos de retirada}

Enquanto os delineamentos de sonda visam avaliar a generalização dos efeitos das VIs para outras VDs, os delineamentos de retirada tentam avaliar a generalização dos efeitos das VIs ao longo do tempo. Nestes delineamentos, a condição experimental não é retirada totalmente, de modo abrupto. O objetivo principal não é demonstrar o efeito inicial de uma condição experimental, mas verificar se ele se mantém em condições diferentes - o que é importante especialmente em pesquisas aplicadas (Kazdin, 1982). Diferentes partes ou componentes de uma condição experimental podem ser retirados gradualmente, um de cada vez, em seqüência. Desta forma, pode-se avaliar a cada manipulação se há ou não manutenção das alterações mensuradas na VD, antes da retirada total da VI. Outra possibilidade pode ser aplicada ao se trabalhar com um delineamento de linha de base múltipla, quando a retirada (seja de um componente ou de toda a condição experimental) restringe-se a apenas uma das várias VDs com as quais se esteja trabalhando (isto é, um dos comportamentos, sujeitos ou situações). Dito de outra forma, esta possibilidade envolve a introdução de uma fase de reversão para um dos comportamentos, sujeitos ou situações investigadas. Os resultados desta fase de reversão podem então apontar (mas não garantir) se haverá manutenção, caso se programem fases de reversão para todos os outros comportamentos, sujeitos ou situações. No estudo de Bornstein, Bellack e Hersen (1977), citado anteriormente para ilustrar o delineamento de linha de base múltipla, por exemplo, após o treino de habilidades sociais ter sido aplicado aos três comportamentos-alvo de Jane, os pesquisadores poderiam ter retirado a VI da primeira VD (manter contato visual), introduzindo uma fase de reversão para este comportamento-alvo. Caso o contato visual permanecesse em alta freqüência, a VI poderia então ser retirada das outras VDs (falar em tom de voz audível e solicitar mudança de comportamento do parceiro).

\section{CONSIDERAÇÕES FINAIS}

Muitos delineamentos experimentais podem ser planejados e executados com a combinação de carac- 
terísticas de dois ou mais dos delineamentos discutidos até agora. Nesses casos, o objetivo é sempre aumentar a força da demonstração experimental, isto é, criar mais oportunidades de comparação entre os efeitos da condição experimental e os da condição controle. Tais delineamentos combinados podem ser necessários para lidar com questões que podem dificultar inferências válidas sobre os efeitos da VI em estudos experimentais. Tais questões podem ter sido antecipadas pelo pesquisador antes mesmo da efetiva implementação do experimento, ou podem surgir com o experimento já em andamento, a partir dos resultados parciais obtidos - o que quer dizer que a decisão de utilizar um delineamento combinado pode ser tomada mesmo após o início do experimento. A possibilidade de modificar o delineamento experimental quando este já está em andamento é uma característica importante dos delineamentos de sujeito único e é uma de suas características distintivas, como argumentou Sidman (1960/1976).

A amplitude das possíveis variações de delineamentos combinados não permite que se possa discutir ou ilustrar todas elas. A combinação de delineamentos em um experimento visa aproveitar os benefícios particulares de cada um deles para o estabelecimento de conclusões experimentais. Deve-se atentar, porém, para o fato de que os problemas pertinentes a cada um desses delineamentos também podem se estender ao delineamento combinado que os utilize. Por causa disso, o pesquisador sempre deve pesar os prós e os contras de cada delineamento antes de utilizá-lo.

Por fim, deve-se considerar, em certos casos, a possibilidade de combinação dos delineamentos de sujeito único com os delineamentos entre-grupos. Por um lado, cada um desses delineamentos se adequa melhor a diferentes maneiras de fazer perguntas experimentais (ou colocar problemas de pesquisa). Por outro, certas variáveis podem gerar resultados que conduzem a interpretações distintas sobre seus efeitos quando investigadas com cada um desses delineamentos. A avaliação do efeito de um medicamento, por exemplo, quando realizada por meio de um delineamento entre-grupos poderia indicar a eficácia do medicamento (para $75 \%$ dos sujeitos do grupo experimental), enquanto por meio de um delineamento de sujeito único poderia indicar que o medicamento não é eficaz (para os 8 sujeitos obesos estudados). Isto se daria, neste exemplo, pela dificuldade dos delineamentos entre-grupos de captar como a VI interage com VEs específicas atuando sobre cada indivíduo (por ex., a obesidade dos sujeitos do experimento de sujeito único). Além disso, certas situações aplicadas podem permitir a utilização de apenas um desses delineamentos. A investigação de problemas médicos raros, por exemplo, pode exigir o uso de delineamentos de sujeito único. Tudo isso deve ser considerado ao se escolher qual delineamento utilizar ao se realizar uma pesquisa específica (Kazdin, 1982).

\section{Questões de Estudo}

1) Defina com suas próprias palavras o que é um experimento.

2) Cite os três objetivos da experimentação.

3) Um experimento busca estabelecer relações sistemáticas entre variáveis. Estas variáveis são divididas em duas categorias principais. Cite-as e explique cada uma delas.

4) No exemplo dado sobre o experimento de refração da luz de Newton, quais variáveis podem ser classificadas como VIs e qual pode ser classificada como VD?

5) Explique o que são VEs (variáveis estranhas).

6) O que significa delinear um experimento?

7) Defina condição controle e condição experimental.

8) Diferencie delineamento de sujeito único e entre-grupos em relação a: como são planejadas as condições experimentais, como são realizadas as medidas de interesse e como as medidas são comparadas.

9) Que argumentos são utilizados para defender a utilização do delineamento de sujeito único para o estudo do comportamento?

10) Explique porque a estratégia dos estados estáveis é indispensável em um delineamento de sujeito único. Cite três razões.

11) Construa uma tabela comparando os delineamentos de reversão, de linha de base múltipla e de mudança de critério quanto a: definição, vantagens, possíveis variações, e limitações (condições nas quais o delineamento pode não ser adequado).

12) Qual a importância de um delineamento de sonda?

13) Qual o objetivo dos delineamentos de retirada? Com que objetivo pode-se planejá-lo junto a um delineamento de linha de base múltipla?

14) É possível executar delineamentos combinados. Qual a função dessa combinação? Que cuidados devem ser tomados? 


\section{REFERÊNCIAS}

Borstein, M. R., Bellack, A. S., \& Hersen, M. (1977). Social-skills training for unassertive children: A multiple-baseline analysis. Journal of Applied Behavior Analysis, 10, 183-195.

Crease, R. P. (2006). Os dez mais belos experimentos cientificos (M. I. D. Estrada, Trad.). Rio de Janeiro, RJ: Jorge Zahar.

De Luca, R. V., \& Holborn, S. W. (1992). Effects of a variableratio reinforcement schedule with changing criteria on exercise in obese and nonobese boys. Journal of Applied Behavior Analysis, 25, 671-679.

Estes, W. K., \& Skinner, B. F. (1941). Some quantitative properties of anxiety. Journal of Experimental Psychology, 29, 390400.

Ferster, C. B., \& Skinner, B. F. (1957). Schedules of reinforcement. New York, NY: Appleton-Century-Crofts.

Glenn, S. S., \& Field, D. P. (1994). Functions of the environment in behavioral evolution. The Behavior Analyst, 17, 241-259.

Johnston, J. M., \& Pennypacker, H. S. (1993a). Strategies and tactics of behavioral research ( $2^{\mathrm{a}}$ ed.). Hillsdale, NJ: Lawrence Erlbaum.

Johnston, J. M., \& Pennypacker, H. S. (1993b). Readings for strategies and tactics of behavioral research $\left(2^{\mathrm{a}} \mathrm{ed}\right.$.). Hillsdale, NJ: Lawrence Erlbaum.

Kazdin, A. E. (1982). Single-case research designs. New York, NY: Oxford University Press.
Kerlinger, F. N. (1973). Foundations of behavioral research $\left(2^{\mathrm{a}}\right.$ ed.). New York, NY: Holt, Rinehart and Winston.

Kidder, L. H. (Org.) (1987). Selltiz, Wrightsman e Cook: Métodos de pesquisa nas relações sociais ( $2^{\mathrm{a}}$ ed. brasileira) (M. M. H. D’Oliveira, M. M. del Rey, Trad.). São Paulo, SP: EPU.

Matos, M. A. (1990). Controle experimental e controle estatístico: A filosofia do caso único na pesquisa comportamental. Ciência e Cultura, 42, 585-592.

Miller, D. L., \& Kelley, M. L. (1994). The use of goal setting and contingency contracting for improving children's homework performance. Journal of Applied Behavior Analysis, 27, 73-84.

Sidman, M. (1976). Táticas da pesquisa científica: Avaliação dos dados experimentais na psicologia (M. E. Paiva, Trad.). São Paulo, SP: Brasiliense. (Original publicado em 1960)

Skinner, B. F. (1938). The behavior of organisms: An experimental analysis. New York, NY: Appleton-Century-Crofts.

Skinner, B. F. (1947). "Superstition" in the pigeon. The American Psychologist, 2, 426.

Skinner, B. F. (1956). A case history in scientific method. The American Psychologist, 11, 221-233.

Recebido: $23 / 10 / 2007$

Última revisão: $13 / 05 / 2008$ Aceite final: 25/05/2008

Notas:

${ }^{1}$ Os termos causa e relação causal serão utilizados neste artigo de maneira ampla para referir relações observadas ou presumidas entre eventos que estariam envolvidas na constituição do fenômeno observado ou produzido.

${ }^{2}$ Matos (1990) faz uma importante discussão sobre os rótulos variáveis dependentes e independentes e propõe em seu lugar os termos variáveis observadas, ou sob observação, e variáveis experimentais, ou manipuladas. Neste artigo serão utilizados os termos variável dependente e independente por conta de seu uso generalizado.

${ }^{3}$ Ou participantes, respondentes etc., a depender do tipo de pesquisa.

${ }^{4}$ No caso de trabalharmos com comportamento, sempre medimos algum aspecto, ou dimensão, de um ou mais comportamentos, que muitas vezes chamamos de desempenho. Ou seja, em estudos comportamentais, a variável dependente (VD) sempre se refere a comportamento. Eventualmente, quando impossibilitados de medir diretamente o comportamento medimos efeitos dos comportamentos e, nestes casos, são necessários cuidados metodológicos especiais (Johnston \& Pennypacker, 1993a, 1993b).

${ }^{5}$ Muitas vezes se faz uma distinção entre os termos "resposta" - que seria a ação/atividade do organismo - e "comportamento" - que seria a relação sujeito-ambiente. Outras vezes essa distinção não é vista como relevante (Glenn \& Field, 1994). Nos "delineamentos de linha de base múltipla entre comportamentos" mais freqüentemente se manipulam condições ambientais que afetam respostas, ou seja, manipula-se o ambiente e mensuram-se seus efeitos sobre as atividades dos sujeitos. No entanto, outras vezes manipula-se o ambiente e mensura-se o efeito sobre classes de respostas - relações sujeito-ambiente já estabelecidas. Neste artigo utilizam-se os termos resposta e comportamento como se fossem intercambiáveis.

${ }^{6}$ Nos relatos de pesquisa, algumas vezes o rótulo "condição" é utilizado para se referir aos diferentes conjuntos de VIs e VDs enquanto o termo "fase" é utilizado para se referir aos distintos parâmetros de um mesmo conjunto de VIs ou valores de VDs. Outras vezes, os termos são utilizados inversamente. No presente artigo, são usados como sinônimos e, por esta razão, apresenta-se os termos "subfases" ou "subcondições", que também são encontrados na literatura experimental. 
Sobre os autores:

Angelo Augusto Silva Sampaio: Mestrando em Psicologia Experimental: Análise do Comportamento (PUC-SP). Bolsista CNPq. Endereço eletrônico: angsampaio@uol.com.br.

Flávia Henriques Baião de Azevedo: Mestranda em Psicologia Experimental: Análise do Comportamento (PUC-SP). Bolsista FAPESP. Endereço eletrônico: flavia_hba@hotmail.com.

Luciana Roberta Donola Cardoso: Mestranda em Psicologia Experimental: Análise do Comportamento (PUC-SP). Endereço eletrônico: lucidonola@uol.com.br.

Camila de Lima: Mestranda em Psicologia Experimental: Análise do Comportamento (PUC-SP). Bolsista CAPES. Endereço eletrônico: camila.campelia@gmail.com.

Mateus Brasileiro Reis Pereira: Mestrando em Psicologia Experimental: Análise do Comportamento (PUC-SP). Bolsista FAPESP. Endereço eletrônico: mateus_brasileiro@yahoo.com.br.

Maria Amalia Pie Abib Andery: Doutora em Psicologia Social (PUC-SP). Professora titular da PUC-SP. CNPq - Produtividade em Pesquisa. 